\title{
Ordering in spatially anisotropic triangular antiferromagnets
}

\author{
Oleg A. Starykh ${ }^{1}$ and Leon Balents ${ }^{2}$ \\ ${ }^{1}$ Department of Physics, University of Utah, Salt Lake City, UT 84112 \\ ${ }^{2}$ Department of Physics, University of California, Santa Barbara, CA 93106-4030
}

(Dated: September 25, 2018)

\begin{abstract}
We investigate the phase diagram of the anisotropic spin- $1 / 2$ triangular lattice antiferromagnet, with interchain diagonal exchange $J^{\prime}$ much weaker than the intrachain exchange $J$. We find that fluctuations lead to a competition between (commensurate) collinear antiferromagnetic and (zigzag) dimer orders. Both states differ in symmetry from the spiral order known to occur for larger $J^{\prime}$, and are therefore separated by quantum phase transitions from it. The zero-field collinear antiferromagnet is succeeded in a magnetic field by magnetically-ordered spin-density-wave and cone phases, before reaching the fully polarized state. Implications for the anisotropic triangular magnet $\mathrm{Cs}_{2} \mathrm{CuCl}_{4}$ are discussed.
\end{abstract}

PACS numbers: $75.10 . J m, 75.50 . \mathrm{Ee}$

The nearest-neighbor spin- $1 / 2$ Heisenberg antiferromagnet on the two dimensional triangular (hexagonal) lattice has long been a prototypical theoretical model for frustrated quantum magnetism [1]. A simple and relevant generalization is the nearest-neighbor spatially anisotropic triangular antiferromagnet, defined by

$$
H=\frac{1}{2} \sum_{i j} J_{i j} \vec{S}_{i} \cdot \vec{S}_{j}-h \sum_{i} S_{i}^{z}
$$

Here $i, j$ are sites on the triangular lattice, $J_{i j}=J, J^{\prime}$ along horizontal and diagonal links, respectively, and zero otherwise (see Fig. 1). The quasi-one-dimensional (1D) inorganic salt, $\mathrm{Cs}_{2} \mathrm{CuCl}_{4}[2]$, with $J^{\prime} / J=0.34$, provides an experimental application. Considerable theoretical interest in $\mathrm{Cs}_{2} \mathrm{CuCl}_{4}$ was spurred by the observation of a strong inelastic continuum in neutron scattering $[3]$, prompting many interesting two-dimensional spin-liquidbased interpretations [4, 5, 6]. There are also very detailed studies of the magnetization process and associated temperature $(T)$ vs magnetic field $(h)$ phase diagram [2, 7]. Existing quantitative comparisons with experiments are based on semiclassical spin-wave calculations [8, 9, 10]. This well-studied scheme cannot, however, be applied to the very interesting (and, we argue below, experimentally relevant) $1 \mathrm{~d}$ limit of $J^{\prime} \ll J$, where the system is best described as a collection of weakly coupled quantum-critical spin- $1 / 2$ chains.

In this Letter, we report a systematic analysis of this important limit, as a function of magnetic field, including the interesting case of zero field. This is accomplished using a Renormalization Group (RG) analysis of the perturbations, represented by the interchain exchange $J^{\prime}$, to the exactly solved problem of decoupled Heisenberg chains. As the RG progresses, new interchain couplings, consistent with symmetries of the lattice model (11), are generated. According to standard RG arguments, the low energy physics is controlled by the couplings which renormalize to dimensionless values of order one first. We find that in zero field all dominant inter-

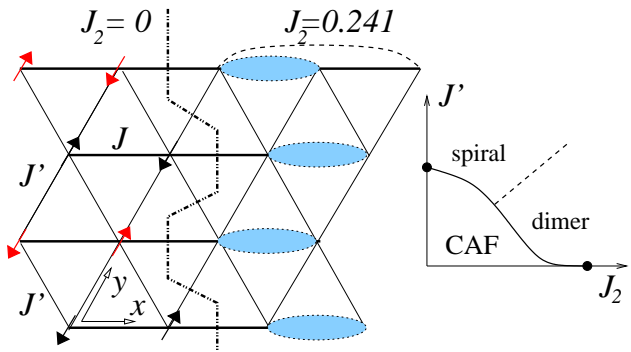

FIG. 1: (color online) Left: The triangular lattice, showing $\left(J, J^{\prime}, J_{2}\right)$ exchange interactions, and integer coordinates $x, y$. Black (red or gray) arrows indicate spin order in the CAF phase on odd (even) chains $\left(J_{2}=0\right.$ section). Solid ovals show the strong bonds in the ordered zig-zag dimer state $\left(J_{2}=0.241\right.$ section $)$. Right: suggested zero-field phase diagram (solid points indicate position of phase boundaries on the axes).

chain couplings are generated by fluctuations, and missed in a naïve analysis. The fluctuation-generated relevant interactions foster two competing orders: collinear antiferromagnetism $(\mathrm{CAF})$ and spontaneous dimerization. For the simple Heisenberg model, this competition is resolved by a marginal intra-chain interaction, in favor of the CAF state. A more general picture, illustrated in Fig. 1, emerges in the presence of an additional secondneighbor exchange $J_{2}$ along the chain axis, exposing the competing (four-fold degenerate) spontaneously dimerized zig-zag state. In a magnetic field, we recover the main low energy features of the $\mathrm{Cs}_{2} \mathrm{CuCl}_{4}$ experiments.

We begin with the zero field case. The low energy properties of each decoupled Heisenberg chain are described by a critical Wess-Zumino-Novikov-Witten (WZNW) $\mathrm{SU}(2)_{1}$ theory, with central charge $c=1$. The low energy theory is connected to the lattice model by the continuum limits of the spin and exchange operators:

$$
\begin{aligned}
\vec{S}_{x, y} & \rightarrow \vec{M}_{y}(x)+(-1)^{x} \vec{N}_{y}(x), \\
\vec{S}_{x, y} \cdot \vec{S}_{x+1, y} & \rightarrow(-1)^{x} \varepsilon_{y}(x) .
\end{aligned}
$$


The dominant fluctuations of a single chain are evident above in the operators $N_{y}^{a}(x)$ and $\varepsilon_{y}(x)$, which both have scaling dimension $1 / 2$ and stronger correlations than any other operators. We have also introduced the the conserved uniform (spin) magnetization density $\vec{M}_{y}$ (scaling dimension 1). In terms of the usual right (R) and left (L) moving chiral spin currents, $\vec{M}_{y}=\vec{J}_{R, y}+\vec{J}_{L, y}$. Here we use the integer coordinates $x, y$ indicated in Fig 1.

Let us now consider the operators which perturb the WZNW theory. This includes a marginal "backscattering" term already for a single Heisenberg chain, and, in addition, terms arising from inter-chain coupling. In unfrustrated systems of coupled chains, e.g. a simple rectangular lattice, a naïve treatment of these latter interactions, that consists in replacing the spin operators in the inter-chain exchange $J^{\prime}$ using Eq.(2), is sufficient [11]. In our case this produces the following perturbation:

$$
\begin{aligned}
H^{\prime}= & \sum_{y} \int d x\left\{\gamma_{\mathrm{bs}} \vec{J}_{R, y} \cdot \vec{J}_{L, y}\right. \\
& \left.+\gamma_{M} \vec{M}_{y} \cdot \vec{M}_{y+1}+\gamma_{\mathrm{tw}} \vec{N}_{y} \cdot \partial_{x} \vec{N}_{y+1}\right\},
\end{aligned}
$$

with $\gamma_{\mathrm{bs}} \approx O(J), \gamma_{M}=2 J^{\prime}, \gamma_{\mathrm{tw}}=J^{\prime}$. The effect of frustration is evident in Eq. (44) by the absence of direct $\vec{N}_{y} \cdot \vec{N}_{y+1}$ coupling between chains. Instead, one obtains only the small residual "twist" term $\gamma_{t w}$, whose scaling dimension is increased by 1 by the spatial derivative. This marginally relevant term leads on its own (for instance in a two-chain zig-zag ladder) to a very weak incommensurate spiral spin order with an exponentially small gap. 12]

However, the naïve interactions in Eq.(4) are incomplete, due to the dynamical generation of strongly relevant interchain interactions. From simple power counting, the most relevant interactions possible should involve products of the $\vec{N}_{y}$ or $\varepsilon_{y}$ operators on different chains. Between nearest-neighbor chains, however, such interactions are forbidden by symmetry under reflection in a plane perpendicular to the chains. However, there is no symmetry prohibiting relevant couplings between second neighbor chains:

$$
H^{\prime \prime}=\sum_{y} \int d x\left\{g_{N} \vec{N}_{y} \cdot \vec{N}_{y+2}+g_{\varepsilon} \varepsilon_{y} \varepsilon_{y+2}\right\} .
$$

The terms $g_{N}, g_{\varepsilon}$ have scaling dimension 1, and if present, are strongly relevant. Being symmetry allowed, one expects them to be generated by fluctuations (in the RG sense) on short scales, similarly to Refs. [13, 14].

A convenient method to derive (5) from (44) is to formally perturbatively integrate out, say, odd chains from the action corresponding to (4). While the resulting even-chain action is naturally non-local, it is conveniently treatable by the RG. At first order in this action, the RG generates a local derivative term $\propto \partial_{x} \vec{N}_{y} \cdot \partial_{x} \vec{N}_{y+2}$. At second order, the RG induces the terms in (5) by more standard calculations very similar to those in
Refs. [13, 14]. This happens already during the initial stage of the RG $\left(0<\ell \lesssim \ell_{0} \sim O(1)\right)$. Because these terms then grow rapidly with increasing $\ell$, further generation of these terms can be neglected. Therefore the effect of fluctuations is to determine "initial conditions":

$$
g_{\varepsilon}\left(\ell_{0}\right)=-\frac{3}{2} g_{N}\left(\ell_{0}\right) \sim \frac{3 A_{0}^{x} \gamma_{M}^{2} \gamma_{\mathrm{tw}}^{2}}{64 \pi v^{3}}=\frac{3 A_{0}^{x}\left(J^{\prime}\right)^{4}}{2 \pi^{4} J^{3}},
$$

using the values of $\gamma_{\mathrm{tw}}, \gamma_{M}$ and velocity $v=\pi J / 2$. Here $A_{0}^{x}$ is a normalization factor for the $\vec{N}$ field [15]. Despite the smallness of $g_{\varepsilon}\left(\ell_{0}\right), g_{N}\left(\ell_{0}\right)$, it is clear that they will dominate the low energy physics, since the scale required for the remaining marginal interactions to grow large, $L_{\text {marg }} \sim \exp \left[c\left(J / J^{\prime}\right)\right]$ is exponentially long.

Naïvely, the competition between $g_{\varepsilon}, g_{N}$ is keen, since the generated values in Eq.(6) are of the same order. However, the outcome is definitely determined by the marginal intra-chain backscattering interaction $\gamma_{\mathrm{bs}}$. This can be seen by considering the coupled RG flow of these three interactions (we neglect terms of $O\left(J^{\prime 6}\right)$ or smaller):

$\partial_{\ell} \tilde{\gamma}_{\mathrm{bs}}=\tilde{\gamma}_{\mathrm{bs}}^{2}, \partial_{\ell} \tilde{g}_{N}=\tilde{g}_{N}-\frac{1}{4} \tilde{\gamma}_{\mathrm{bs}} \tilde{g}_{N}, \partial_{\ell} \tilde{g}_{\varepsilon}=\tilde{g}_{\varepsilon}+\frac{3}{4} \tilde{\gamma}_{\mathrm{bs}} \tilde{g}_{\varepsilon},(7)$

where $\tilde{g}_{X}=g_{X} /(2 \pi v)(X=\mathrm{bs}, N, \varepsilon)$. Here $\tilde{\gamma}_{\mathrm{bs}}(\ell=$ $0) \equiv-\Gamma \approx-0.23<0,[16]$ so the backscattering is marginally irrelevant and flows logarithmically to zero: $\tilde{\gamma}_{\mathrm{bs}}(\ell)=-\Gamma /(1+\Gamma \ell)$. For an isolated Heisenberg chain, $\tilde{\gamma}_{\text {bs }}$ only weakly modifies the low energy behavior by logarithmic corrections [17]. Because $\tilde{\gamma}_{\text {bs }}$ enters the RG equations for $\tilde{g}_{N}, \tilde{g}_{\varepsilon}$ multiplicatively, however, it becomes crucial in the coupled-chains problem. Indeed, from (7) the relevant couplings grow according to

$$
\frac{\tilde{g}_{N}(\ell)}{\tilde{g}_{N}\left(\ell_{0}\right)}=\Xi_{\ell}^{1 / 4} e^{\ell-\ell_{0}}, \quad \frac{\tilde{g}_{\varepsilon}(\ell)}{\tilde{g}_{\varepsilon}\left(\ell_{0}\right)}=\Xi_{\ell}^{-3 / 4} e^{\ell-\ell_{0}},
$$

where $\Xi_{\ell}=(1+\Gamma \ell) /\left(1+\Gamma \ell_{0}\right)$. With the small initial values in Eq.(6), the relevant couplings become of order one at the scale $\ell^{*} \sim 4 \ln \left(J / J^{\prime}\right) \gg 1$. Then since $\Gamma \ell^{*} \gg 1$ we have $\frac{\tilde{g}_{N}\left(\ell^{*}\right)}{\tilde{g}_{\varepsilon}\left(\ell^{*}\right)}=\Xi_{\ell} \gg 1$. Thus backscattering parametrically enhances the antiferromagnetic interaction between second neighbor chains over the competing dimerization one. As a result, subsystems of even and odd chains order into Néel patterns independently of each other on the scale $\ell^{*}$, when $\tilde{g}_{N}\left(\ell^{*}\right) \approx 1$. The remaining coupling $\gamma_{M}$ between these two rectangular Néel sublattices remains small and can still be treated perturbatively. By a standard quasi-classical spin wave calculation, appropriate for the magnetically ordered Néel sublattices, one observes the familiar phenomenon of order-from-disorder, which locks the two sublattices into the collinear CAF phase shown in Fig. 1. For values of $J^{\prime} \approx J$, it is known that the ground state is a rather different spiral, smoothly deformed from the famous $120^{\circ}$ order obtaining for $J^{\prime}=J[18]$. These two phases differ in symmetry, and must be separated by at least one quantum phase transition (which can be $1^{\text {st }}$ order) when $J^{\prime} / J \sim O(1)$. 
Now consider the effect of an additional secondneighbor antiferromagnetic exchange along the chains, $J_{2}$. This is well-known to decrease the initial value of the backscattering, $\Gamma$. In particular, $\Gamma \rightarrow 0$ at the critical value $J_{2}^{\text {crit }}=0.241$ [16], which separates the critical (Luttinger) phase of the spin chain from a spontaneously dimerized one. With non-zero $J^{\prime}$, sufficiently close to this critical point one finds $\Gamma \ell^{*} \sim 1$, and the backscatteringinduced enhancement of $g_{N}$ is eliminated. From Eq.(6), the larger initial value of $\left|g_{\varepsilon}\right|$ induces instead a twodimensional dimerization instability! Moreover, comparing the RG scales of the relevant, $\ell^{*}$, and backscattering, $\ell_{\mathrm{bs}}=1 / \Gamma$, terms in the vicinity of the $J_{2}^{\text {crit }}$ critical point, we deduce the phase boundary between the collinear and dimer phases: $J^{\prime} /\left.J\right|_{\text {C-D }} \sim \exp \left[-0.11 J /\left(J_{2}^{\text {crit }}-J_{2}\right)\right]$. Symmetry again requires at least one additional phase boundary (dashed in Fig. 11) between the spiral and dimerized phases. The nature of the transitions in Fig 1 is beyond the scope of this paper.

Let us turn now to the situation in non-zero field. It is convenient to work at fixed magnetization, $M=$ $\frac{1}{N} \sum_{i} S_{i}^{z}=\frac{1}{N} \sum_{y} \int d x M_{y}^{z}(x)$, rather than fixed field. The 1d Heisenberg model retains $c=1$ free-boson character for any $0<M<1 / 2$, and can be viewed as an "easy-plane" deformation of the WZNW model. All scaling dimensions can be expressed in terms of the "boson radius" $R$, which is a known function of $M$ [19]. For $0<M<1 / 2$, the transverse XY components of the staggered magnetization field $N^{ \pm}=N^{x} \pm i N^{y}$ strengthen their correlations, and have scaling dimension $\Delta_{\mathrm{xy}}=\pi R^{2}$ decreasing from $1 / 2$ at $M=0$ toward $1 / 4$ at $M=1 / 2^{-}$. This is in accordance with the semiclassical canted XY antiferromagnetic order in a field. Conversely, the staggered magnetization along the field direction shifts to the incommensurate wavevectors $\pi \pm 2 \delta$,

$$
N_{y}^{z}(x) \rightarrow \mathcal{S}_{y}^{z+} e^{i 2 \delta x}+\mathcal{S}_{y}^{z-} e^{-i 2 \delta x}
$$

with $\delta=\pi M$. The correlations at this wavevector progressively weaken as $M$ is increased, such that $\mathcal{S}_{y}^{z \pm}$ has scaling dimension $\Delta_{z}=\left(4 \pi R^{2}\right)^{-1}$ increasing from $1 / 2$ toward 1 as $M \rightarrow 1 / 2^{-}$. The dimerization operator $\varepsilon_{y}$ is expressible as a different linear combination of $\mathcal{S}_{y}^{z \pm}$ and is not an independent degree of freedom for $M \neq 0$. Finally, the zero field magnetization operator develops an expectation value: $M_{y}^{z}(x) \rightarrow M+\delta M_{y}^{z}(x)$, and the fluctuation piece $\delta M_{y}^{z}(x)$ has scaling dimension 1 as before.

These changes in the correlations have several effects. The shift of the longitudinal spin correlations to the incommensurate wavevector $\pi \pm 2 \delta$ removes the frustration of the longitudinal piece of the inter-chain $J^{\prime}$ spin interaction. The Z-components of neighboring spins on a chain are no longer antiparallel, and the effective exchange field exerted by them upon the shared spin on the adjacent chain does not cancel. This leads to a relevant term

$$
H_{\mathrm{sdw}}^{\prime}=\sum_{y} \int d x \gamma_{\mathrm{sdw}} \mathcal{S}_{y}^{z+} \mathcal{S}_{y+1}^{z-}+\text { h.c. }
$$

with $\gamma_{\mathrm{sdw}}=\left(1-e^{2 i \delta}\right) J^{\prime}$ non-zero for $M>0$. For small magnetization, this term is almost as relevant as the fluctuation-generated terms in Eq.(5), but its magnitude is $O\left(J^{\prime} \delta\right)$ and so is significantly larger for $\delta \gtrsim\left(J^{\prime} / J\right)^{3}$. For field above $h_{1} \approx 3 A_{0}^{x} /(8 \pi)\left(J^{\prime} / J\right)^{3} h_{\text {sat }}$ it becomes the dominant instability channel, and stabilizes a longitudinal "spin density wave" (SDW) with the incommensurate wavevector $k_{x}=\pi \pm 2 \delta$ and all spin expectation values aligned along the field. However, as $M$ is further increased, its scaling dimension $\Delta_{\mathrm{SDw}}=\left(2 \pi R^{2}\right)^{-1}$ becomes less relevant, approaching 2 at the saturation.

Simultaneously, the XY piece of the twist term,

$$
H_{\mathrm{tw}}^{\prime}=\sum_{y} \int d x \frac{\gamma_{\mathrm{tw}}}{2}\left(N_{y}^{+} \partial_{x} N_{y+1}^{-}+\text {h.c. }\right) \text {, }
$$

becomes increasingly more relevant with $M$ (as compared to marginal in zero field). Its scaling dimension, $\Delta_{\mathrm{tw}}=2 \pi R^{2}+1$, decreases steadily from 2 toward $3 / 2$ as $M \rightarrow 1 / 2^{-}$. The transition between two ordered states takes place when the two scaling dimensions become approximately equal at $\pi R^{2}=(\sqrt{5}-1) / 4 \approx 0.309$, which corresponds to $M \approx 0.3$ (see Figures 8 and 9 in Ref. 19). Thus for $M \gtrsim 0.3$, the twist term dominates and induces a "cone" state, in which the XY components of the spins spiral at a wavevector close to but not equal to $k_{x}=\pi$. One may wonder whether the fluctuationgenerated terms in (5), particularly the transverse piece of $g_{N}$, can intervene between the two described states. However, because it is only generated at fourth order in $J^{\prime}$, we find that it is always subdominant to either $\gamma_{\mathrm{sdw}}$ or $\gamma_{\mathrm{tw}}$, at all fields. The resulting phase diagram of $J-J^{\prime}$ model in magnetic field is sketched in Figure 2 .

We now apply this understanding to $\mathrm{Cs}_{2} \mathrm{CuCl}_{4}$. We first address an objection to the quasi-1d approach, raised in Ref. 7, that the magnetization curve $M(h)$ of $\mathrm{Cs}_{2} \mathrm{CuCl}_{4}$ differs significantly from that of the $1 \mathrm{~d}$ Heisenberg chain. In fact, very good agreement can be obtained simply by assuming that spins are completely uncorrelated between different chains. By calculating the expectation value in a direct product state on different chains, the ground state energy suffers a correction obtained by simply replacing $J^{\prime} \vec{S}_{i} \cdot \vec{S}_{j} \rightarrow J^{\prime} M^{2}$ for each $J^{\prime}$-link. Using $h=-\partial E / \partial M$ and the known thermodynamics of decoupled Heisenberg chains, one obtains the curve in Fig. 2 , Thus the observed $M(h)$ curve in fact indicates the weakness of inter-chain spin correlations in $\mathrm{Cs}_{2} \mathrm{CuCl}_{4}$. However, by its definition, $M(h)$ depends only upon $\left\langle\vec{S}_{i} \cdot \vec{S}_{j}\right\rangle$ for nearest-neighbor $i, j$. This explains how both spinwave theory 7, [8] and our quasi-1d approach, which differ drastically in longer-range correlations, can reproduce the experimental $M(h)$ curve. 

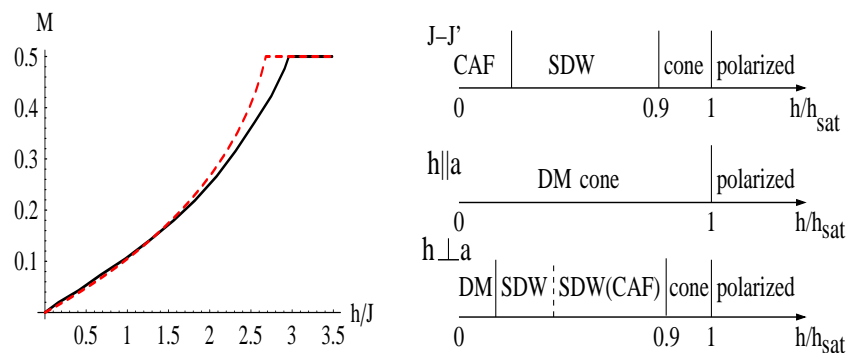

FIG. 2: (color online) Left panel: Comparison of experimental (solid black line - obtained by scanning Fig.2a, $B \| a$, of Ref. 7]) and theoretical (dashed red line) magnetization curves for $\mathrm{Cs}_{2} \mathrm{CuCl}_{4}, J^{\prime} / J=0.34$. The experimental (and theoretical) $M(h)$ is nearly direction-independent apart from $g$-factor rescaling. Right panel: $T=0$ phase diagram of $J-J^{\prime}$ model (top line) and $\mathrm{Cs}_{2} \mathrm{CuCl}_{4}\left(h \| a\right.$ and $h \perp a$ lines). $h_{\text {sat }}$ is the field at which $M=1 / 2$ is reached.

Long-range correlations are crucial for understanding the low-temperature phase diagram of $\mathrm{Cs}_{2} \mathrm{CuCl}_{4}$. An important (and unfortunate!) complication of this material is a significant Dzyaloshinskii-Moriya (DM) coupling along the interchain links. Although nominally small, $D \approx 0.05 J \approx 0.16 J^{\prime}$, it plays a key role because it is non-frustrated. Specifically, applying Eq.(2) gives the inter-chain DM coupling $H_{\mathrm{DM}}=$ $\sum_{y} \int d x 2 D\left(N_{y}^{b} N_{y+1}^{c}-N_{y}^{c} N_{y+1}^{b}\right)$, taking conventional crystalline axes $a, b, c$. In zero field, this term is as relevant as the fluctuation-induced interactions in (6), but is much larger since $D / J \gg\left(J^{\prime} / J\right)^{4}$. Thus it dominates and drives spiral magnetic order, as seen experimentally. Magnetic field along the $a$ axis ( $h \| a$ in Fig. 2h) strengthens $\{b, c\}$ components of $\vec{N}$, and, hence, the DM-cone phase, which extends all way to $h_{\text {sat }}$.

When the field lies in the $b-c$ plane $(h \perp a$ in Fig. (2), the situation is more interesting. In this case, the DM coupling involves both XY and Z components of the $\vec{N}$ field. From (9) we see that the correlations of these two fields become incommensurate. This effectively nullifies the DM term once the incommensurability $2 \delta \gtrsim A_{0}^{x} D / J$. Thus most of the behavior in this field orientation can be understood from the simple $J-J^{\prime}$ model in a field (top line in Fig. 2, right panel) discussed above. The same conclusion was reached in Ref. 8. The experimental phase diagram for $h \perp a$ in Fig 2 of Ref.[7], indeed shows evidence of the expected DM, SDW, and cone phases. Moreover, in neutron measurements 2], the ordering wavevector is consistent with the $\delta=\pi M$ expected for the SDW state for $H=1-2 T$, and evidences the cone state near $h_{\text {sat. }}$.

At fields intermediate between these two limits, however, experiment in addition observes one or two (dependent upon field orientation in the $b-c$ plane) commensurate ordered states, 7, 20] denoted as SDW(CAF) in
Fig. 2. An explanation within the $J-J^{\prime}$ model was proposed in Ref. 20, based upon an extrapolation of the spinflip expansion about the fully-polarized state. Some partial confirmation of this notion is found in our approach: the symmetry of the observed order is consistent with what would be induced by the fluctuation-induced $g_{N}<0$ interaction in a field. [21] However our calculations above indicate that the magnitude of $g_{N}$ is much too low to explain the experimental ordering temperature $T_{c}(h)$ [7] (note there are no logarithmic enhancements analogous to those in Eqs. (8) in large fields). We instead suggest that a relatively small direct antiferromagnetic exchange interaction $J_{2}^{\prime}$ between spins on second-neighbor chains is a more probable explanation. We find only $J_{2}^{\prime} \approx 0.06 \mathrm{~J}$ is needed to explain the flat $T_{c}(h)$ curve [7].

The authors acknowledge insightful discussions with R. Coldea, F. Essler and T. Senthil, and support from NSF DMR04-57440 (L. B.) the Packard foundation (L. B.), and from ACS PRF 43219-AC10 (O. S.).

[1] P.W. Anderson, Mater. Res. Bull. 8, 153 (1973).

[2] R. Coldea et al., Phys. Rev. Lett. 86, 1335 (2001).

[3] R. Coldea, D. A. Tennant, and Z. Tylczynski, Phys. Rev. B 68, 134424 (2003).

[4] C.H. Chung, K. Voelker, and Y.B. Kim, Phys. Rev. B 68, 094412 (2003).

[5] S.V. Isakov, T. Senthil, and Y.B. Kim, Phys. Rev. B 72, 174417 (2005).

[6] J. Alicea, O.I. Motrunich, and M.P.A. Fisher, Phys. Rev. Lett. 95, 247203 (2005).

[7] Y. Tokiwa et al., Phys. Rev. B 73, 134414 (2006).

[8] M.Y. Veillette, J.T. Chalker, and R. Coldea, Phys. Rev. B 71, 214426 (2005).

[9] M.Y. Veillette, A.J.A. James, and F.H.L. Essler, Phys. Rev. B 72, 134429(2005).

[10] D. Dalidovich et al., Phys. Rev. B 73, 184403 (2006).

[11] H. J. Schulz, Phys. Rev. Lett. 77, 2790 (1996).

[12] A.A. Nersesyan, A.O. Gogolin, and F.H.L. Essler, Phys. Rev. Lett. 81, 910 (1998).

[13] O.A. Starykh and L. Balents, Phys. Rev. Lett. 93, 127202 (2004).

[14] O.A. Starykh, A. Furusaki, and L. Balents, Phys. Rev. B 72, 094416 (2005).

[15] T. Hikihara and A. Furusaki, Phys. Rev. B 69, 064427 (2004).

[16] S. Eggert, Phys. Rev. B 54, R9612 (1996).

[17] M. Bocquet et al., Phys. Rev. B 64, 094425 (2001).

[18] W. Zheng, R.H. McKenzie, and R.R.P. Singh, Phys. Rev. B 59, 14367 (1999).

[19] I. Affleck and M. Oshikawa, Phys. Rev. B 60, 1038 (1999).

[20] M.Y. Veillette and J.T. Chalker, Phys. Rev. B 74, 052402 (2006).

[21] L. Balents and O.A. Starykh, unpublished. 